\title{
Emotion Expression of Robot with Personality
}

\author{
Xue Hu, ${ }^{1,2}$ Lun Xie, ${ }^{1,2}$ Xin Liu, ${ }^{2,3}$ and Zhiliang Wang ${ }^{1,2}$ \\ ${ }^{1}$ School of Computer and Communication Engineering, University of Science and Technology Beijing, Beijing 100083, China \\ ${ }^{2}$ Beijing Key Laboratory of Knowledge Engineering for Materials Science, Beijing 100083, China \\ ${ }^{3}$ School of Automation and Electrical Engineering, University of Science and Technology Beijing, Beijing 100083, China
}

Correspondence should be addressed to Lun Xie; xielun@ustb.edu.cn

Received 19 April 2013; Accepted 8 July 2013

Academic Editor: Vishal Bhatnagar

Copyright (C) 2013 Xue Hu et al. This is an open access article distributed under the Creative Commons Attribution License, which permits unrestricted use, distribution, and reproduction in any medium, provided the original work is properly cited.

\begin{abstract}
A robot emotional expression model based on Hidden Markov Model (HMM) is built to enable robots which have different personalities to response in a more satisfactory emotional level. Gross emotion regulation theory and Five Factors Model (FFM) which are the theoretical basis are firstly described. And then the importance of the personality effect on the emotion expression process is proposed, and how to make the effect quantization is discussed. After that, the algorithm of HMM is used to describe the process of emotional state transition and expression, and the performance transferring probability affected by personality is calculated. At last, the algorithm model is simulated and applied in a robot platform. The results prove that the emotional expression model can acquire humanlike expressions and improve the human-computer interaction.
\end{abstract}

\section{Introduction}

In the studies of psychology, emotion is a state which is aroused and experienced by the individual constantly. The aroused process can be either conscious or unconscious. The emotional response sometimes is affected by the environment, but sometimes it comes into contradictions and conflicts and is incompatible with a specific situations; thus, it is necessary for the individuals to adapt to the environment using emotional regulation [1]. Emotion regulation is a process that individuals manage and change the generation and expression of emotions by strategies. Thompson thought that "Emotional regulation may be defined as the extrinsic and intrinsic processes responsible for monitoring, evaluating, and modifying emotional reactions, especially their intensive and temporal features" [2]. Gross thought emotion regulation meant the individuals influenced what kind of emotions they would have, when to have them, and how to experiment and express them [3].

With the development of artificial psychology, robotics, and computer technology, robots with different functions gradually change people's work and life and help human beings to engage in complex and complicated manual labor. In addition to the intelligent features, mental activities like emotion, personality, willing, and creation are necessary to robots. Thus, emotion regulation is important to robots in human-computer interaction. Lazarus and Folkman proposed the way of coping paradigm and distinguished the emotion regulation between problem-focused copingpersons' attempts to change the situation or get rid of menaces caused by tension, typically by using problemsolving strategies-and emotion-focused coping-persons' attempts to lessen emotional distress by engaging behavioral or cognitive regulation strategies [4]. It regarded emotion regulation as a one-off act. Two-stage process model of emotion regulation proposed by Gross thought it expanded in the emotional process, which meant different strategies were used in different stages [1]. All these studies ignored the effect of the individual personality characteristics on the process of emotional generation and expression. Some researchers described the process of emotional state transition by the mathematical model of Finite State Machine (FSM), which included the influence of individual personality and emotional stimulus [5]. Some others applied backfeed loop [6], 
B-model [7], nonparametric cumulative sum (CUSUM) [8], and et cetera to changing the cognitive and expression of emotion. However, something about how to quantify the personality characteristic parameter and how the personality influences the emotional expression in the process of emotion regulation have not been studied clearly.

In this paper, we firstly describe Gross' emotion regulation process briefly and analyze the influence of emotional expression strategy over emotional behaviors. Then, based on HMM, we quantify the Gross' process model of emotion regulation and expound the transfer and expression of emotional states. According to the Five Factor Model scales, the effect of personality on expression suppression strategy is calculated in HMM. Finally, the mathematic model of robot with personality is built and applied in the human-computer interaction situation.

\section{Gross' Process Model of Emotion Regulation and the Five Factor Model}

2.1. Gross Model of Emotion Regulation. Different strategies are used in different stages of emotion generation. There are two parts of emotional regulation in the Gross' model: antecedent-focused emotion regulation and response-focused emotion regulation. The antecedentfocused emotion regulation is divided into four different types of strategies: situation selection, situation modification, attentional deployment, and cognitive change. And the fifth strategy which is named as response modulation is a response-focused strategy. Figure 1 shows an overview of these strategies.

Situation selection is the first antecedent-focused emotion regulation strategy in Gross' model. It refers to the fact that individuals unconsciously approach or avoid certain events or occasions to regulate their own emotions. The second antecedent-focused emotion regulation strategy in the model is situation modification, which is an attempt to control and adjust uncomfortable emotional events in the environment. The third antecedent-focused emotion regulation strategy is attentional deployment, which means individuals choose one or more attentional focuses in many aspects of the situation. The fourth antecedent-focused emotion regulation strategy is cognitive change that refers to selecting the possible meanings to the emotional events. Different cognitive meaning may generate different emotion states. The fifth emotion regulation strategy, response modulation, a response-focused strategy, happens after emotion arousal and affects emotional responses like psychological experience, behavioral expression, and physiological responses.

According to Gross' model, there are many emotional regulation strategies in the process of emotion generation. The most common and useful strategies are reappraisal and suppression. Reappraisal, which is an antecedent-focused emotion regulation strategy, changes individual's cognitive understanding of emotional events so as to change emotional experience. Suppression is a response-focused strategy and is used after emotional generation. Individuals use this strategy to change tendency of emotional reaction, mainly including expressions and physiological responses. This strategy mobilizes the individual's self-control, but it only regulates the expression of emotions and external behavior, and the emotional experience has not changed. Individuals can control their emotions better with a stronger capability of self-control. In the same situation, individuals with different personalities may have different emotional behavior. This paper focuses on how suppression strategy influences emotion expression and what is the effect of personality.

2.2. Model of Personality. Personality is one's responses of thinking, emotion, and behavior, influenced by heredity and environment. In a period of time, it has the character of stability. One's personality has an effect on the process of emotional generation, reappraisal, and expression [9]. In psychological research, the Five Factor Model (FFM) [10] which is also named as the OCEAN Model is one of the most recent models proposed so far. The FFM contains five basic dimensions: openness, conscientiousness, extraversion, agreeableness, and neuroticism. The trait of openness describes a person's cognitive style. Conscientiousness means the way we control, manage and regulate our own impulse. The extraversion trait represents the number and density of human interaction, the need for stimulation, and the ability obtaining pleasure. Agreeableness examines the individual's attitude to others, and neuroticism reflects individual's process of emotion regulation, the tendency of negative emotion experience, and the instability of emotion. Each trait is represented by one dimension, and thus, the FFM is a five-dimension model. The five-element vector $\mathbf{P}=\left[\begin{array}{lllll}p_{1} & p_{2} & p_{3} & p_{4} & p_{5}\end{array}\right]$ refers to one's personality, and $p_{i}, i=1,2, \ldots, 5$ separately stands for the value of openness, conscientiousness, extraversion, agreeableness, and neuroticism. The effect of personality on suppression strategy in Gross' emotion regulation model is mainly discussed in this paper, and the weight of effect under each personality trait is calculated by Analytic Hierarchy Process (AHP).

2.3. Expression Parameter of Personality Suppression. Individual's personality traits are evaluated by NEO-PI-R scales, and personalized vector $\mathbf{P}=\left[\begin{array}{lllll}p_{1} & p_{2} & p_{3} & p_{4} & p_{5}\end{array}\right]$ could be obtained. The five factors have different effects on suppression, and AHP is used to calculate the effect weight $w_{1}, w_{2}, w_{3}, w_{4}, w_{5}$, from which we can obtain weight vector $\mathbf{W}=\left[\begin{array}{lllll}w_{1} & w_{2} & w_{3} & w_{4} & w_{5}\end{array}\right]^{T}$.

(a) Firstly building simplified hierarchical model as shown in Figure 2. This simplified model contains two layers: destination and criteria. Suppression strategy is the destination, and traits in FFM are the criteria.

(b) Constructing the pairwise comparison matrix $A$. The comparative value between trait $p_{i}$ and $p_{j}$ is shown in Table 1. The bigger the value is, the more influential $p_{i}$ is compared with $p_{j}$ to the suppression of emotion expression. 


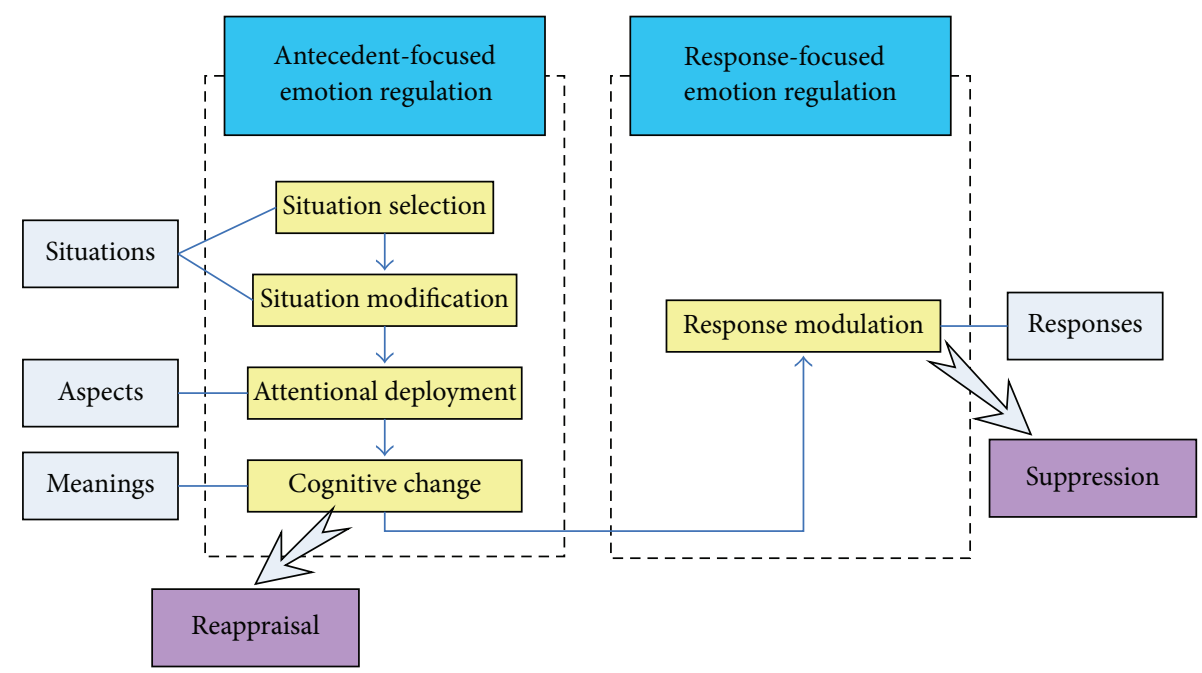

FIGURE 1: Strategies of Gross emotion regulation model.

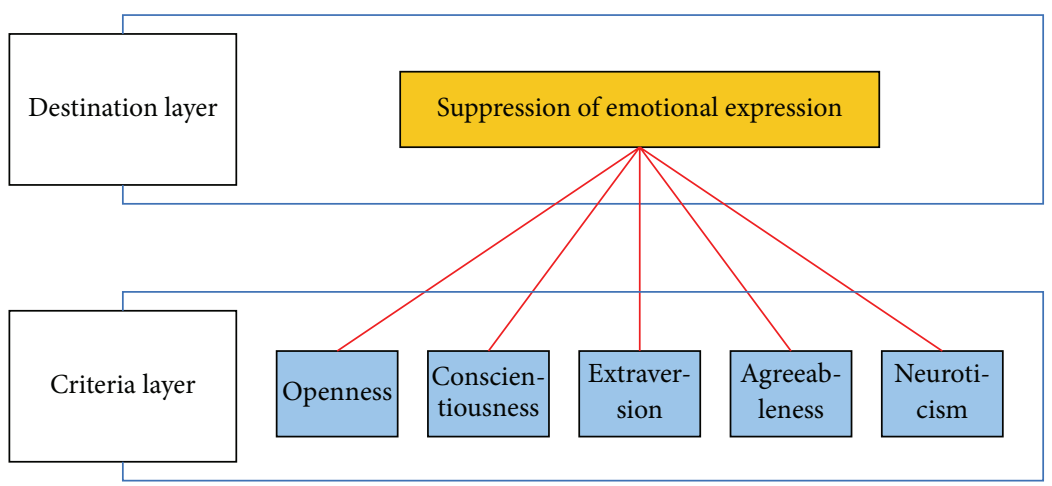

FIgURE 2: The simplified hierarchical model.

Then, the comparison matrix $A$ can be obtained as follows and $a_{j i}=1 / a_{i j}$ :

$$
\mathbf{A}=\left(a_{i j}\right)_{5 \times 5}=\left(\begin{array}{ccccc}
1 & 5 & \frac{1}{5} & 3 & \frac{1}{3} \\
\frac{1}{5} & 1 & \frac{1}{9} & \frac{1}{3} & \frac{1}{7} \\
5 & 9 & 1 & 7 & 3 \\
\frac{1}{3} & 3 & \frac{1}{7} & 1 & \frac{1}{5} \\
3 & 7 & \frac{1}{3} & 5 & 1
\end{array}\right)
$$

Normalizing $A$ for each column vector by the following formula, $A^{\prime}$ is obtained:

$$
\begin{gathered}
a_{i j}^{\prime}=\frac{a_{i j}}{\sum_{i=1}^{5} a_{i j}}, \quad(i=1,2, \ldots, 5, j=1,2, \ldots, 5) \\
\mathbf{A}^{\prime}=\left(a_{i j}^{\prime}\right)_{5 \times 5}=\left(\begin{array}{lllll}
0.105 & 0.200 & 0.112 & 0.184 & 0.071 \\
0.021 & 0.040 & 0.062 & 0.020 & 0.031 \\
0.524 & 0.360 & 0.560 & 0.429 & 0.642 \\
0.035 & 0.120 & 0.080 & 0.061 & 0.043 \\
0.312 & 0.280 & 0.187 & 0.306 & 0.214
\end{array}\right)
\end{gathered}
$$

$\widetilde{w}_{i}=\sum_{j=1}^{5} a_{i j}^{\prime}$ is calculated by summing $a_{i j}^{\prime}$ in row, and $w_{i}$ is normalized by the following formula:

$$
w_{i}=\frac{\widetilde{w}_{i}}{\sum_{i=1}^{5} \widetilde{w}_{i}}=\frac{\sum_{j=1}^{5} a_{i j}^{\prime}}{\sum_{i=1}^{5} \sum_{j=1}^{5} a_{i j}^{\prime}} .
$$

Finally, the weight vector can be gained: $\mathbf{W}=$ $\left[\begin{array}{lllll}0.134 & 0.035 & 0.503 & 0.068 & 0.260\end{array}\right]^{T}$.

The ability suppressed by personality in the process of emotional expression is defined as follows:

$$
\begin{gathered}
E=\mathbf{P} \times \mathbf{W}=\sum_{i=1}^{5} p_{i} \cdot w_{i}, \\
\eta=\frac{E}{\sum_{i=1}^{5} p_{i}} .
\end{gathered}
$$

$\eta$ is the expression parameter of personality suppression. 
TABLE 1: The comparative value between traits.

\begin{tabular}{|c|c|c|c|c|c|}
\hline $\begin{array}{l}\text { Suppression of } \\
\text { emotion expression }\end{array}$ & Openness & Conscientiousness & Extraversion & Agreeableness & Neuroticism \\
\hline Openness & 1 & 5 & $1 / 5$ & 3 & $1 / 3$ \\
\hline Conscientiousness & $1 / 5$ & 1 & $1 / 9$ & $1 / 3$ & $1 / 7$ \\
\hline Extraversion & 5 & 9 & 1 & 7 & 3 \\
\hline Agreeableness & $1 / 3$ & 3 & $1 / 7$ & 1 & $1 / 5$ \\
\hline Neuroticism & 3 & 7 & $1 / 3$ & 5 & 1 \\
\hline
\end{tabular}

\section{Emotion Regulation Model Based on HMM and the Effect of Personality on Emotion Expression}

3.1. Gross' Emotion Regulation Model Based on HMM. The emotional process includes generation of emotion and expression of behavior. Emotional arousal is a random process, which is not only in connection with the current stimulus but also the emotional state of the individual. Behavioral expression is a random process of emotional expression after psychological experience generated. The two kinds of random process are in line with HMM, and thus we can use it to describe Gross' process model of emotion regulation in a mathematical method $[5,11]$.

HMM is a statistical probability model including hidden states and Markov chain that is represented by parameters. As shown in Figure 3, HMM is a double stochastic process that consists of a Markov chain and a general stochastic process.

Markov chain is used to describe the transfer of emotional states by the transition probability. And the general stochastic process is used to describe the relationship between the emotional states and the observed sequence by the observation probability. The process of states transition cannot be observed, thus the model is named as "hidden" Markov model.

HMM consists of two-state sets and three probability matrices.

(a) Hidden state set $S=\left[S_{1}, S_{2}, \ldots, S_{N}\right]$ : these states, that meet the Markov properties, usually cannot be obtained through direct observation.

(b) Observation state set $O=\left[O_{1}, O_{2}, \ldots, O_{M}\right]$ : it is a state set associated with hidden states, and its state number can be different from the hidden state set's.

(c) Initial state probability matrix $\pi=\left[p_{1}, p_{2}, \ldots, p_{N}\right]$, $\sum_{i=1}^{N} p_{i}=1$ : it refers to the probability distribution of hidden state in the initial moment.

(d) Hidden state transition probability matrix $\mathbf{A}=$ $\left(a_{i j}\right)_{N \times N}, \sum_{j=1}^{N} a_{i j}=1$ : it describes the transition probabilities between the hidden states in HMM.

(e) Observation state transition probability matrix $\mathbf{B}=$ $\left(b_{j k}\right)_{N \times M}, \sum_{k=1}^{M} b_{j k}=1$ : it represents the probability that when hidden state is $S_{j}$, the observed state is $O_{k}$, here, $b_{j k}=p\left(O_{k} \mid S_{j}\right), 1 \leq j \leq N, 1 \leq k \leq M$.

Currently, emotion classification models used commonly in human-computer interaction are the OCC model [12] which specifies 22 categories for emotions, the PAD model [13] that contains three dimensions of pleasure, arousal, and dominance, and the basic emotion model identified by Ekman [14] which includes happiness, anger, disgust, fear, sadness, and surprise. The robot's emotional state set is defined as $S=\left[S_{1}, S_{2}, \ldots, S_{N}\right] . N$ is the number of emotional states, and $q_{i}, i=1,2, \ldots, N$ is the probability that state $S_{i}$ is generated, $\sum_{i=1}^{N} q_{i}=1,0 \leq q_{i} \leq 1(i=1,2, \ldots, N)$.

The probability space of emotion state can be regarded as

$$
\left(\begin{array}{c}
S \\
Q
\end{array}\right)=\left(\begin{array}{llll}
S_{1} & S_{2} & \cdots & S_{N} \\
q_{1} & q_{2} & \cdots & q_{N}
\end{array}\right) .
$$

In this paper, in addition to the six basic emotions, calming is used in the robot emotional states, and this means $N=7$.

Expression of human is various, but most psychologists agree that all expression can be divided into six categories: happiness, anger, disgust, fear, sadness, and surprise, corresponding to the six basic emotions, respectively. The emotion of robot can be observed by the expression of its output, which is the observed state in the observation matrix. Its expression is different to basic emotions because of the different intensity of emotion [15]. The six basic emotions are divided into three area of strength: low, mid, and high, so there are three expressions corresponding to each basic emotion. Therefore, the robot has 19 kinds of expressions including calming, and $M=19$.

The HMM emotion model presented in this paper is about the effect of individual's personality on the process of emotion expression, and the emotional inherent performance which reflects the person's emotional variation, has nothing to do with the character of the individual. In human interaction, we will make a description to the interacted one-such as one person is very cheerful and optimistic, or one person is stable and cautious-obviously it is very difficult for a robot to describe a person with his own characters. In the paper, we assume that the emotion state of a robot has been generated, which means the hidden state has come out by Markov chain, and personality influences the expression of emotion state. Assuming that the robot is in a certain initial state, and the initial probability distribution is $\boldsymbol{\pi}_{0}$, the emotional state probability distribution changes under the hidden state transition probability matrix $\mathbf{A}$ and generates the hidden state $S_{j}$; using the suppression strategy, the output of expression $O_{k}$ can be obtained by the observation state transition probability matrix $\mathbf{B}^{\prime}$ which is corrected by the personality suppression of expression parameter $\eta$. 


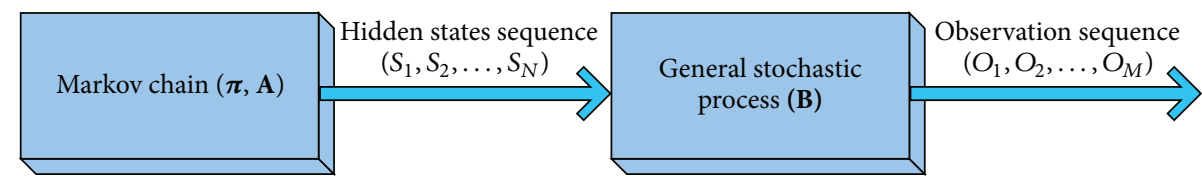

Figure 3: Structure of HMM.

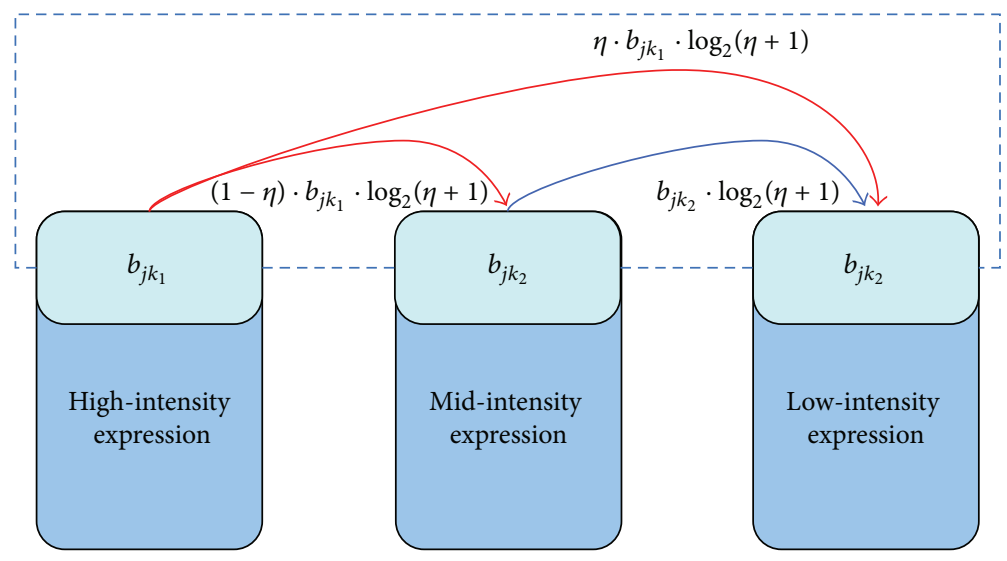

FIGURE 4: The change of $b_{j k}$ by the personality suppression of expression parameter $\eta$.

3.2. Effect of Personality on Observation State Transition Probability Matrix. It will get an emotional state called hidden state $S$ which is recorded as $t+1$, after emotional state is updated by Markov chain. Robots make certain emotional behavior according to that an emotional state and pass it to emotional expression system. To a certain particular emotional status, its expression is different by its inner character. For instance, when it happens to a same happy state, to those open and active personality individuals, the facial expression is much more obvious and vivid compared to those introversion and sensitive personality individuals. So after gaining individual's emotional state, we still need one random process to calculate expression output, which is the general random process in HMM.

The observation state transition probability matrix $\mathbf{B}$ refers to the probability of 7 kinds of expressions under 7 emotions:

$$
\mathbf{B}=\left(b_{j k}\right)_{7 \times 7}=\left(\begin{array}{cccc}
b_{11} & b_{12} & \cdots & b_{17} \\
b_{21} & b_{22} & \cdots & b_{27} \\
\vdots & \vdots & \ddots & \vdots \\
b_{71} & b_{72} & \cdots & b_{77}
\end{array}\right), \quad \sum_{k=1}^{7} b_{j k}=1
$$

Expect for calming, the other 6 expressions are divided into high-intensity, mid-intensity, and low-intensity expression. Thus $\mathbf{B}$, is a $7 \times 19$ matrix:

$$
\mathbf{B}=\left(b_{j k_{l}}\right)_{7 \times 19}=\left(\begin{array}{cccccc}
b_{11_{1}} & b_{11_{2}} & b_{11_{3}} & b_{12_{1}} & \cdots & b_{17} \\
b_{21_{1}} & b_{21_{2}} & b_{21_{3}} & b_{22_{1}} & \cdots & b_{27} \\
\vdots & \vdots & \vdots & \vdots & \ddots & \vdots \\
b_{71_{1}} & b_{71_{2}} & b_{71_{3}} & b_{72_{1}} & \cdots & b_{77}
\end{array}\right)
$$

$$
\sum_{k=1}^{6} \sum_{l=1}^{3}\left(b_{j k_{l}}+b_{j 7}\right)=1 .
$$

Here, $l$ refers to intensity of emotion, and $l=1, l=2$, $l=3$ represents separately high-intensity, mid-intensity, and low-intensity expressions $b_{j k_{l}}(1 \leq j \leq 7,1 \leq k \leq 6)$ is the probability that $k$ th kind of expression with $l$ th intensity generates under $j$ th emotion, and $b_{j 7}(1 \leq \mathrm{j} \leq 7)$ is the probability of calming under $j$ th emotion.

The effect that personality has on suppression strategy performs in external expression which refers to the observation state transition probability matrix $\mathbf{B}$. In the paper, $b_{j k}$ is changed by the effect of the personality suppression of expression parameter $\eta$ as shown in Figure 4.

In Figure 4, the red arrow indicates the sum of transition probability from high-intensity expression to mid-intensity and low-intensity expressions which is $b_{j k_{1}} \cdot \log _{2}(\eta+1)$, including the probability $(1-\eta) \cdot b_{j k_{1}} \cdot \log _{2}(\eta+1)$ from high- to mid-intensity expression and $\eta \cdot b_{j k_{2}} \cdot \log _{2}(\eta+1)$ from high- to low-intensity expression. This indicates that with bigger $\eta$, the transition probability from high- to mid-intensity expression is smaller, and the transition probability from high- to lowintensity expression is bigger. That is, the stronger the ability of self-control is, the more possible the low-intensity expression happens. On the contrary, with smaller personality suppression of expression parameter $\eta$, the sum of transition probability from high-intensity expression to mid-intensity and low-intensity expression is smaller. That is, the weaker the ability of self-control is, the more closed the expression is to the inner experience. The blue arrow indicates the probability from mid- to low-intensity expression, and the meaning is when the personality suppression of expression parameter $\eta$ is bigger, low-intensity expression performs more possibly. 
The mathematic equations can be derived as follows:

$b_{j k_{l}}^{\prime}$

$$
=\left\{\begin{aligned}
b_{j k_{1}}^{\prime}= & b_{j k_{1}}-b_{j k_{1}} \cdot \log _{2}(\eta+1) \\
b_{j k_{2}}^{\prime}= & b_{j k_{2}}+(1-\eta) \cdot b_{j k_{1}} \cdot \log _{2}(\eta+1) \\
& -b_{j k_{2}} \cdot \log _{2}(\eta+1) \\
b_{j k_{3}}^{\prime}= & b_{j k_{3}}+\eta \cdot b_{j k_{1}} \cdot \log _{2}(\eta+1)+b_{j k_{2}} \cdot \log _{2}(\eta+1) .
\end{aligned}\right.
$$

Here, $1 \leq j \leq 7,1 \leq k \leq 6$, and the probability of calming has not changed, $b_{j 7}^{\prime}=b_{j 7}$.

It can be proved that

$$
\begin{aligned}
& \sum_{k=1}^{7} b_{j k}^{\prime}=\sum_{k=1}^{6} \sum_{l=1}^{3} b_{j k_{l}}^{\prime}+b_{j 7}^{\prime}=\sum_{k=1}^{6} b_{j k_{1}}^{\prime}+\sum_{k=1}^{6} b_{j k_{2}}^{\prime}+\sum_{k=1}^{6} b_{j k_{3}}^{\prime}+b_{j 7}^{\prime} \\
& =\sum_{k=1}^{6}\left(b_{j k_{1}}-b_{j k_{1}} \cdot \log _{2}(\eta+1)\right) \\
& +\sum_{k=1}^{6}\left[b_{j k_{2}}+(1-\eta) \cdot b_{j k_{1}} \cdot \log _{2}(\eta+1)\right. \\
& \left.-b_{j k_{2}} \cdot \log _{2}(\eta+1)\right] \\
& +\sum_{k=1}^{6}\left[b_{j k_{3}}+\eta \cdot b_{j k_{1}} \cdot \log _{2}(\eta+1)\right. \\
& \left.+b_{j k_{2}} \cdot \log _{2}(\eta+1)\right]+b_{j 7} \\
& =\sum_{k=1}^{6}\left[b_{j k_{1}}-b_{j k_{1}} \cdot \log _{2}(\eta+1)+b_{j k_{2}}\right. \\
& +(1-\eta) \cdot b_{j k_{1}} \cdot \log _{2}(\eta+1)-b_{j k_{2}} \cdot \log _{2}(\eta+1) \\
& +b_{j k_{3}}+\eta \cdot b_{j k_{1}} \cdot \log _{2}(\eta+1) \\
& \left.+b_{j k_{2}} \cdot \log _{2}(\eta+1)\right]+b_{j 7} \\
& =\sum_{k=1}^{6}\left[b_{j k_{1}}+b_{j k_{2}}+b_{j k_{3}}\right]+b_{j 7} \\
& =\sum_{k=1}^{6} \sum_{l=1}^{3}\left(b_{j k_{l}}+b_{j 7}\right)=1 \text {. }
\end{aligned}
$$

Corrected emotional expression matrix satisfies the constraint: $\sum_{k=1}^{M} b_{j k}=1$. Although this model is proposed in the case of the expression intensity divided into three categories, it also applies to other classification of expression intensity.

\section{Experiment}

4.1. Simulation Results. Ekman's six basic emotions include fear, anger, sadness, disgust, happiness, and surprise. To describe the emotion transfer and expression of the robot, the calming emotion is added in this model. Thus, emotion state set $S=\left\{S_{1}, S_{2}, S_{3}, S_{4}, S_{5}, S_{6}, S_{7}\right\}$, here $S_{1} \sim S_{6}$ represent

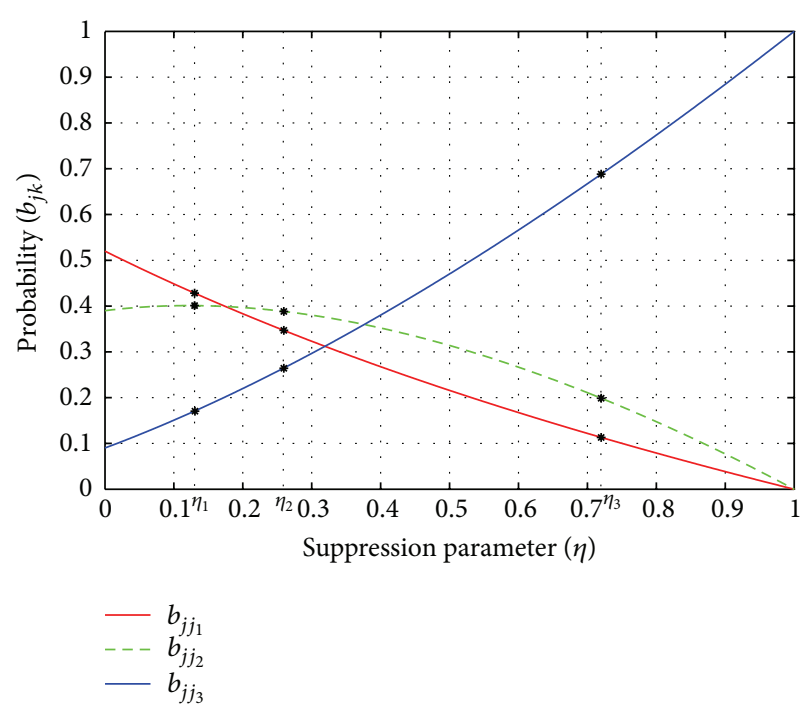

FIGURE 5: The change of probability $b_{j j_{l}}$ under initial probability distribution of sadness $\left[b_{33_{1}}, b_{33_{2}}, b_{33_{3}}\right]=[0.52,0.39,0.09]: b_{j j_{1}}$ decreases, and $b_{j j_{3}}$ increases gradually, and $b_{j j_{2}}$ decreases after increasing a little. To the individual with the personality suppression of expression parameter $\eta_{1}=0.13$, his expression intensity is high, and to the individual with $\eta_{2}=0.26$, his expression is mid intensity, and to the third one with $\eta_{3}=0.72$, the expression is low intensity.

fear, anger, sadness, disgust, happiness, and surprise, and $S_{7}$ is calming. First, we assume that the probability distribution of the hidden state $S=\left[q_{1}, q_{2}, q_{3}, q_{4}, q_{5}, q_{6}, q_{7}\right]$ is known, and the personality vectors $\mathbf{P}_{1}, \mathbf{P}_{2}, \mathbf{P}_{3}$ of three people are obtained by NEO-PI-R scales. Then, the personality suppression of expression parameters $\eta_{1}, \eta_{2}, \eta_{3}$ is calculated by formula (6). The observation state transition probability matrix $\mathbf{B}$ is corrected by $\eta_{1}, \eta_{2}, \eta_{3}$, respectively, and the correction results of the expression occurrence probability in different intensity of different individuals are simulated. Assuming that to a certain hidden state $S_{j}$, only the expression corresponding to the emotion is generated, $j=k$. Here, Figures 5, 6, and 7 are the simulation results of sadness expression $(k=3)$ correction probability.

Figures 5-7 show that with the increase of the personality suppression of expression parameter, that is, the increase in self-control ability, the probability of generating highintensity expression decreases, and probability of generating low-intensity expression increases. When the emotion is sadness $(j=3)$ and initial probability distribution of sadness is different, different intensity expressions appear to the same person; under the same distribution of initial probability, the expression intensity is also different to individuals with different personalities.

4.2. Experience on the Robot Platform. Expression is the most direct manifestation of human emotional states. In the model of the robot's emotion regulation, HMM is used to realize the suppression strategy in Gross's emotion regulation model. With the same initial probability distribution, robots with different personalities have different intensity expressions; 
TABLE 2: Robot's expressions in different intensities.

\begin{tabular}{|c|c|c|c|}
\hline & Low-intensity & Mid-intensity & High-intensity \\
\hline Happines & & & \\
\hline \multicolumn{4}{|l|}{ Sadness } \\
\hline \multicolumn{4}{|l|}{ Fear } \\
\hline \multicolumn{4}{|l|}{ Anger } \\
\hline \multicolumn{4}{|l|}{ Surprise } \\
\hline \multicolumn{4}{|l|}{ Disgust } \\
\hline Calming & & & \\
\hline
\end{tabular}

TABLE 3: The expression results of robots.

\begin{tabular}{lcr}
\hline Hidden state & Robot without personality & Robot with personality \\
\hline Happiness & High-intensity expression & Mid-intensity expression \\
Fear & High-intensity expression & Low-intensity expression \\
Disgust & Mid-intensity expression & Low-intensity expression \\
Sadness & High-intensity expression & Mid-intensity expression \\
Surprise & Mid-intensity expression & Mid-intensity expression \\
Anger & High-intensity expression & Low-intensity expression \\
\hline
\end{tabular}

robots with same personality may have different expression outputs because of different initial probability distributions. In Table 2, different intensity expressions of robot are shown.

In order to verify the actual moderating effects of the model, we apply the personality suppression model on the robot platform and obtain different outputs of expressions using suppression strategy. Three experiences are done in this paper to prove the validity of the model.

4.2.1. Experience 1. In this experience, the observation state transition probability matrix $\mathbf{B}$ is fixed. The expression results of robots with and without personality are recorded in Table 3. The results show that comparing with the robot without personality, the expression of the robot which has personalities is suppressed and that is more in line with the human communication.

4.2.2. Experience 2. The observation state transition probability matrix $\mathbf{B}$ and the hidden state $S$ are fixed in the experience. Five groups of personality vectors are inputted to the robot emotion regulation system. Each group contains 100 different personality vectors, and the five groups have high value in the factor of openness, conscientiousness, extraversion, agreeableness, and neuroticism separately. The statistic expression results of robots with different personalities are recorded as shown in Table 4 . It can be inferred from the results that the extraversion trait in personality influences the expression intensity more than other traits, and 
TABLE 4: Different expression results of robots with different personalities.

\begin{tabular}{lccc}
\hline Personality trait & $\begin{array}{c}\text { High-intensity } \\
\text { expression }\end{array}$ & $\begin{array}{c}\text { Mid-intensity } \\
\text { expression }\end{array}$ & $\begin{array}{c}\text { Low-intensity } \\
\text { expression }\end{array}$ \\
\hline Openness & $19 \%$ & $44 \%$ & $37 \%$ \\
Conscientiousness & $53 \%$ & $35 \%$ & $12 \%$ \\
Extraversion & $6 \%$ & $19 \%$ & $75 \%$ \\
Agreeableness & $39 \%$ & $33 \%$ & $28 \%$ \\
Neuroticism & $17 \%$ & $39 \%$ & $44 \%$ \\
\hline
\end{tabular}

TABLE 5: Outputs of robots with different observation state transition probability matrices.

\begin{tabular}{lccc}
\hline Maximum in $\left[b_{j j_{1}}, b_{j j_{2}}, b_{j j_{3}}\right]$ & Personality vector $\mathbf{P}_{\mathbf{1}}$ & Personality vector $\mathbf{P}_{\mathbf{2}}$ & Personality vector $\mathbf{P}_{\mathbf{3}}$ \\
\hline$b_{j j_{1}}$ & High-intensity & Mid-intensity & Low-intensity \\
& expression & expression & expression \\
$b_{j j_{2}}$ & Mid-intensity & Low-intensity & Low-intensity \\
& expression & expression & expression \\
$b_{j j_{3}}$ & Low-intensity & Low-intensity & Low-intensity \\
& expression & expression & expression \\
\hline
\end{tabular}

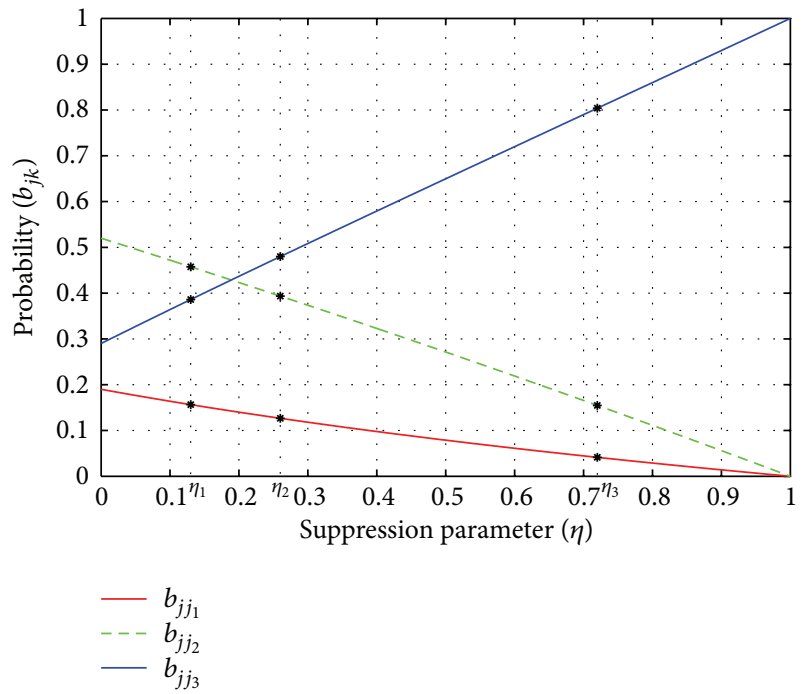

FIGURE 6: The change of probability $b_{j j_{l}}$ under initial probability distribution of sadness $\left[b_{33_{1}}, b_{33_{2}}, b_{33_{3}}\right]=[0.19,0.52,0.29]: b_{j j_{1}}$ decreases more slowly than $b_{j j_{2}}$, and $b_{j j_{3}}$ increases gradually. To the individual with the personality suppression of expression parameter $\eta_{1}=0.13$, his expression intensity is mid, and to the individual with $\eta_{2}=0.26$ and the third one with $\eta_{3}=0.72$, the expression is low intensity.

the conclusion conforms to the point proposed by Gross and John [9].

4.2.3. Experience 3. In experience 3, the hidden state $S$ is fixed, and there are three different personality vectors. The robots' outputs with different observation state transition probability matrices are recorded in Table 5 . The results show that to the same robot with personality, the expression is different because of the different observation state transition probability matrices.

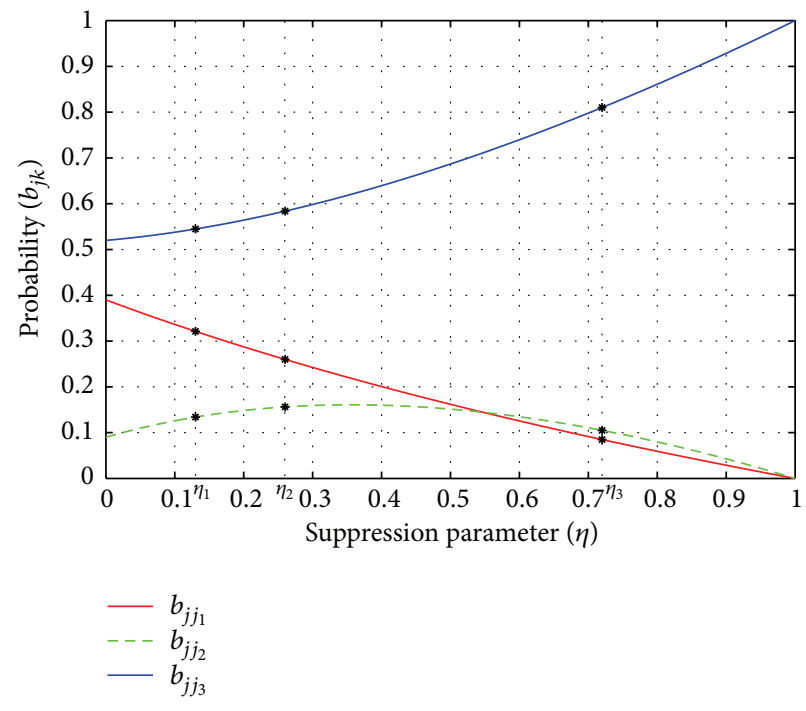

FIGURE 7: The change of probability $b_{j j_{l}}$ under initial probability distribution of sadness $\left[b_{33_{1}}, b_{33_{2}}, b_{33_{3}}\right]=[0.39,0.09,0.52]: b_{j j_{1}}$ decreases, and $b_{j j_{3}}$ increases gradually, and $b_{j j_{2}}$ decreases after increasing a little. To the individual with the personality suppression of expression parameter $\eta_{1}=0.13$, with $\eta_{2}=0.26$ and with $\eta_{3}=$ 0.72 , the expression is low intensity.

Figure 8 shows the typical emotional expressions of the robot. From the three experiences, it can be indicated that the emotional expression suppression model with personality is consistent with the simulation results basically. That means the model proposed in this paper is validated, and humancomputer interaction can be improved by using the model.

\section{Conclusion}

Firstly, this paper analyses the importance of the robot's emotional regulation strateg, and applies the Gross' emotion 


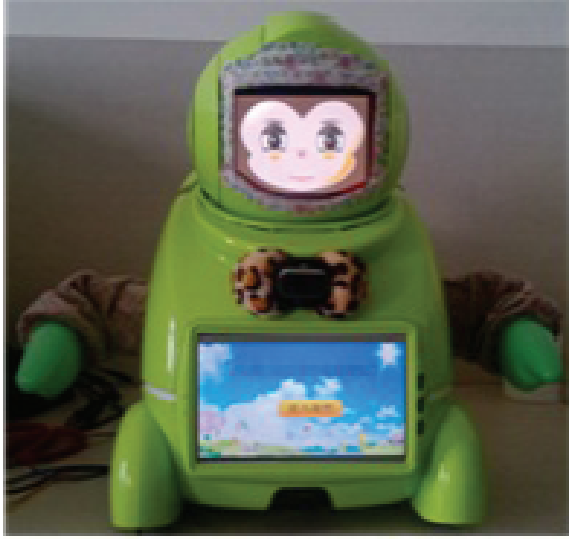

Expression of calming

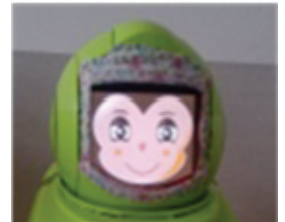

Low-intensity expression of happiness

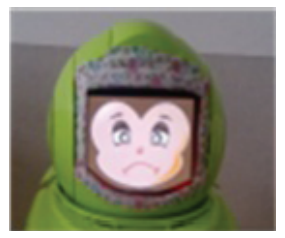

Low-intensity expression of sadness

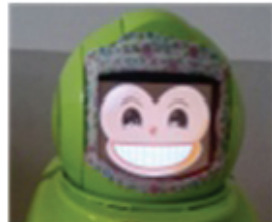

Mid-intensity expression of happiness

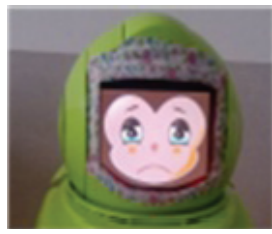

Mid-intensity expression of sadness

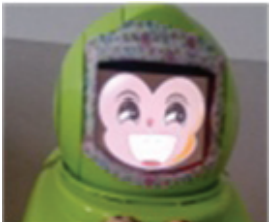

High-intensity expression of happiness

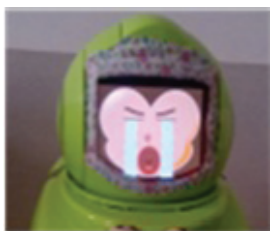

High-intensity expression of sadness

FIgURE 8: Typical emotional expressions of the robot.

regulation model as the psychological basis of robot emotional model. Using HMM algorithm, we deal with the psychological problems mathematically and establish the robot's emotional regulation model. Aiming at the expressional suppression strategy in the process of emotional regulation, we bring in robot personality as an impact factor and then adjust the robot expression matrix. The impact factor does not influence the value of emotional intensity but the occurrence probabilities of different emotional intensities; thus, there is no need to calculate the intensities of different emotions. Using the proposed emotion regulation model in actual robot platform, it can be seen that the robot is more natural and human-like in the human-computer interaction. The statistical analysis of the model analysis and the actual interaction results show that after the introduction of robot personality suppression of expression parameter, anthropomorphic robot is improved, and the effect of human-computer interaction fits the diversity of human emotions.

Because of the sophistication and versatility of human emotion regulation process, many factors affect the regulation. In this paper, we only consider the robot personality, the obvious and influential factor, and cannot fully simulate the human emotion interaction. How to take into account various factors to optimize the model and how to apply the model in the continuous emotional intensity space is one of the main future researches.

\section{Acknowledgments}

This work is supported by National Natural Science Foundation of China (no. 61170115; no. 61170117; no. 61105120), and the 2012 Ladder Plan Project of Beijing Key Laboratory of Knowledge Engineering for Materials Science (no. Z121101002812005).

\section{References}

[1] J. J. Gross, "Emotion regulation: affective, cognitive, and social consequences," Psychophysiology, vol. 39, no. 3, pp. 281-291, 2002.
[2] R. A. Thompson, "Emotional regulation and emotional development," Educational Psychology Review, vol. 3, no. 4, pp. 269$307,1991$.

[3] J. J. Gross, "Emotion regulation: past, present, future," Cognition and Emotion, vol. 13, no. 5, pp. 551-573, 1999.

[4] M. A. Southam-Gerow and P. C. Kendall, "Emotion regulation and understanding: implications for child psychopathology and therapy," Clinical Psychology Review, vol. 22, no. 2, pp. 189-222, 2002.

[5] P. Xiaolan, X. Lun, L. Xin, and W. Zhiliang, "An algorithm model for gross cognitive reappraisal strategy," Advances in Intelligent Systems and Computing, vol. 1, pp. 435-446, 2013.

[6] T. Bosse, M. Pontier, and J. Treur, "A computational model based on Gross' emotion regulation theory," Cognitive Systems Research, vol. 11, no. 3, pp. 211-230, 2010.

[7] A. Soleimani and Z. Kobti, "An adaptive computational model of emotion regulation strategies based on Gross theory," in Proceedings of the 5th Conference on Computer Science \& Software Engineering, pp. 9-17, 2012.

[8] E. Leon, I. Montalban, S. Schlatter, and I. Dorronsoro, "Computer-mediated emotional regulation: detection of emotional changes using non-parametric cumulative sum," in Proceedings of the 32nd Annual International Conference of the IEEE Engineering in Medicine and Biology Society (EMBC '10), pp. 11091112, September 2010.

[9] J. J. Gross and O. P. John, "Individual differences in two emotion regulation processes: implications for affect, relationships, and well-being," Journal of Personality and Social Psychology, vol. 85, no. 2, pp. 348-362, 2003.

[10] R. R. McCrae and O. P. John Jr., "An introduction to the fivefactor model and its applications," Journal of Personality, vol. 60, no. 2, pp. 175-215, 1992.

[11] L. Xin, X. Lun, W. Zhiliang, and F. Dongmei, "Robot emotion and performance regulation based on HMM," International Journal of Advanced Robotic Systems, vol. 10, pp. 1-6, 2013.

[12] L. Pena, J.-M. Pena, and S. Ossowski, "Representing emotion and mood states for virtual agents," in Proceedings of the 9th German Conference on Multi-Agent System Technologies (MATES '11), pp. 181-188, 2011.

[13] J. Woo Park, W. Hyun Kim, W. Hyong Lee, J. Chang Kin, and M. Jin Chung, "How to completely use the PAD space for socially 
interactive robots," in Proceedings of the IEEE International Conference on Robotics and Biomimetics, pp. 3005-3010, 2011.

[14] P. Ekman, "Universals and cultural differences in facial expressions of emotion," in Proceedings of the Nebraska Symposium on Motivation, J. Cole, Ed., vol. 19, pp. 207-282, University of Nebraska Press, Lincoln, Nebraska, 1971.

[15] L. Xie, Z. Wang, and J. Xu, "Mechanical design and affective interaction of bionic robot head," Advanced Science Letters, vol. 4, no. 4-5, pp. 1337-1341, 2011. 


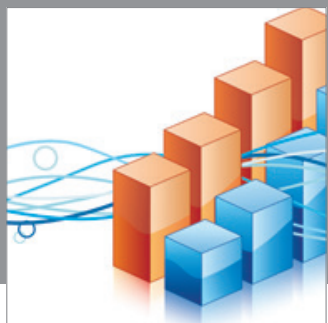

Advances in

Operations Research

mansans

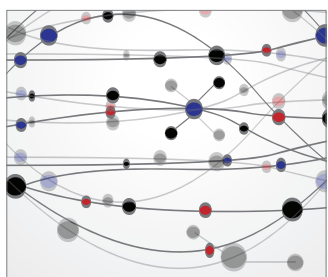

The Scientific World Journal
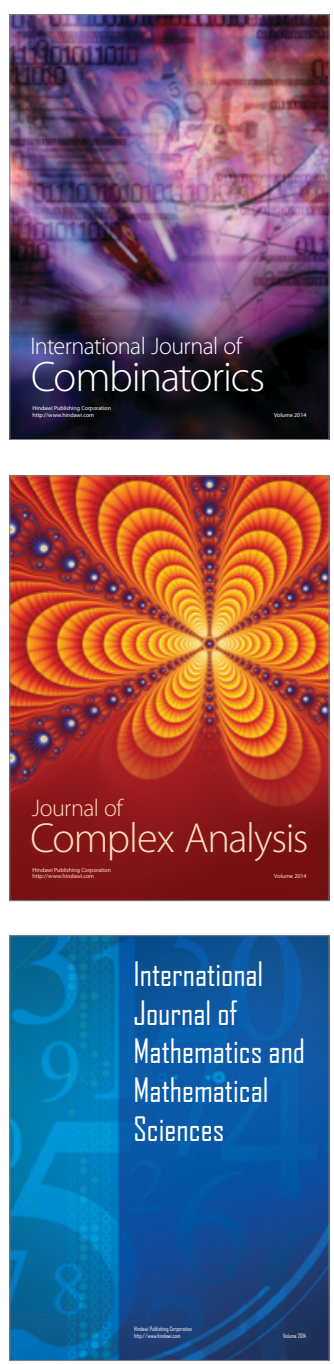
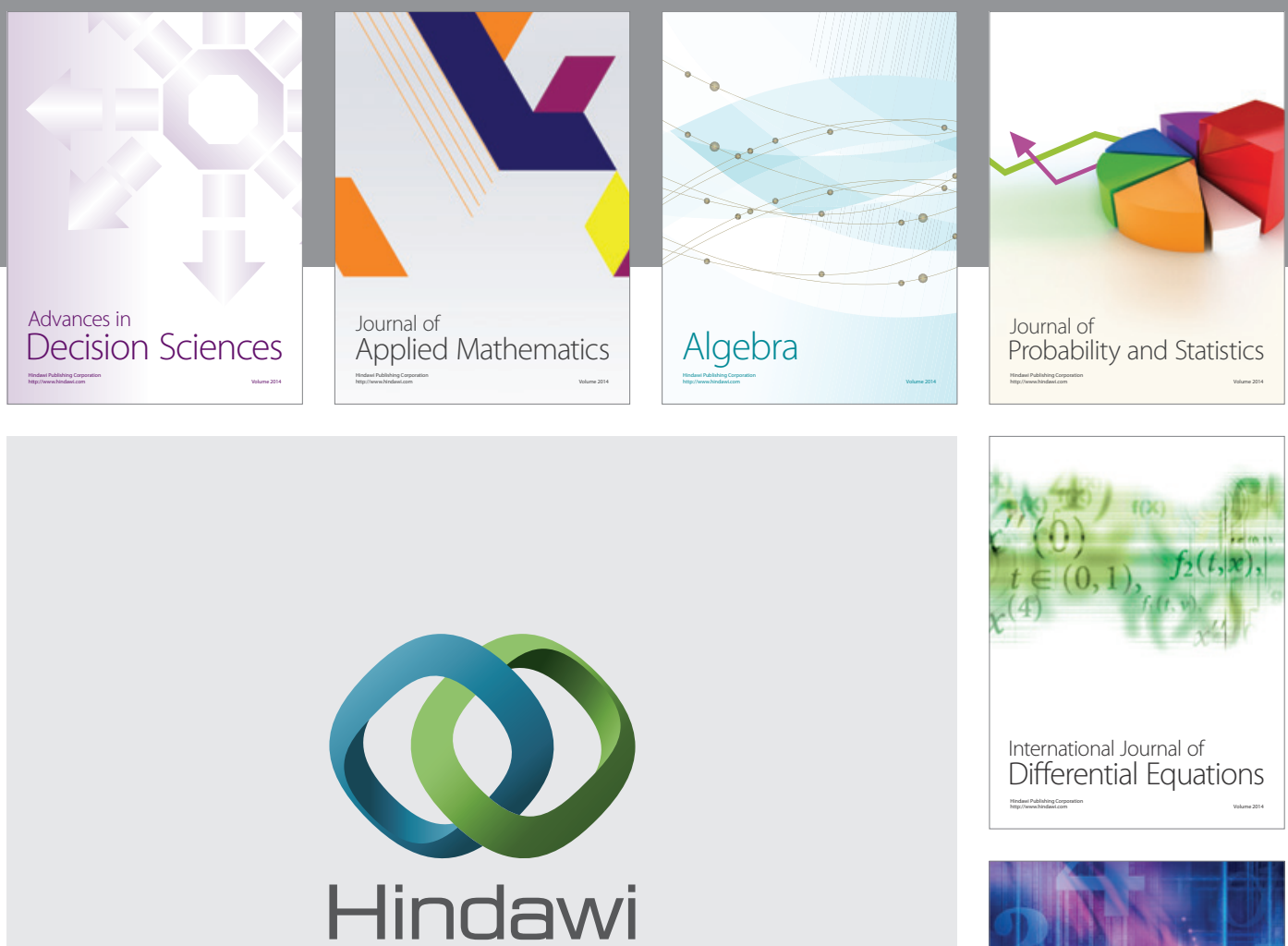

Submit your manuscripts at http://www.hindawi.com
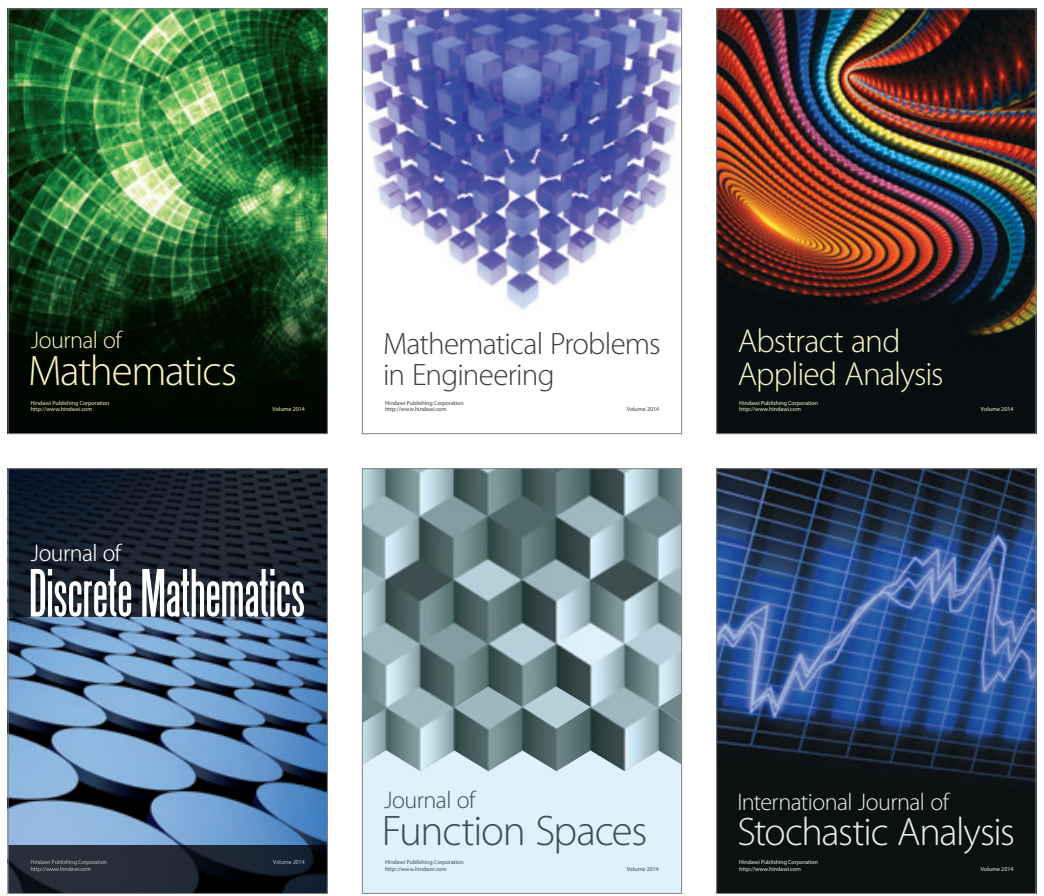

Journal of

Function Spaces

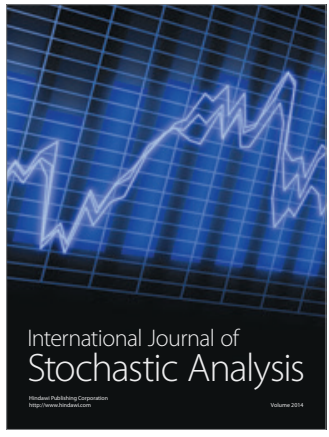

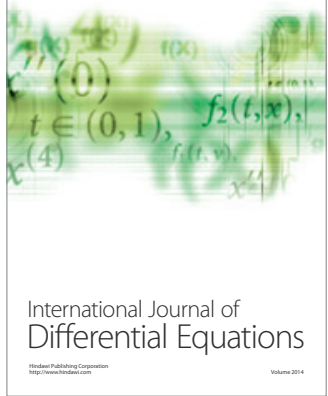
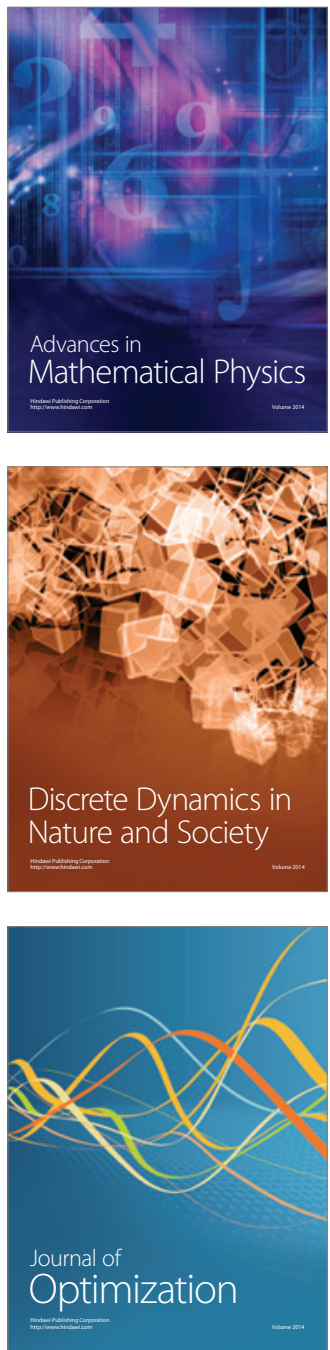